\title{
What is the value of bone remodeling markers in patients with calcium stones?
}

\author{
Miguel Ángel Arrabal-Polo • Salvador Arias-Santiago • \\ Miguel Arrabal-Martin
}

Received: 30 August 2012/ Accepted: 6 September 2012/Published online: 19 September 2012

(C) Springer-Verlag 2012

To the Editor,

The association between recurrent calcium stones and bone mineral density has been studied over years. Patients with recurrent calcium stones shown a decrease in bone mineral density, which is higher in patients with hypercalciuria [1]. Bone mineral density loss produces osteopenia or osteoporosis that can be assessed by bone densitometry or serum values of biochemical markers of bone turnover (bone formation and bone resorption) [2].

In addition to different metabolic and mineral alterations, which are present in patients with calcium stones, such as hypercalciuria, hyperoxaluria or hypocitraturia, patients with recurrent calcium lithogenic activity have shown abnormal values of bone remodeling markers, that in most cases are independent of iPTH activity [3]. Now a days, one of the markers that indicates the activity of bone formation more reliably is osteocalcin, which can be determined in serum and usually shows higher values in patients with recurrent calcium stones [3]. Also, patients with recurrent calcium stones and severe bone resorption present significantly higher serum values $\beta$-crosslaps, a specific marker of bone resorption according to recent studies [3, 4]. This marker indicates a higher osteoclastic activity in patients with calcium stones, and may serve as a follow-up parameter and for medical treatment control (potassium citrate, thiazides or bisphosphonates).

M. Á. Arrabal-Polo $(\bowtie) \cdot$ M. Arrabal-Martin

Section of Urolithiasis, Department of Urology,

San Cecilio University Hospital, Camino de Ronda Street,

143, 4F, 18003 Granada, Spain

e-mail: arrabalp@ono.com

S. Arias-Santiago

Department of Medicine, School of Medicine, Granada, Spain
Our studies on recurrent calcium stones and loss of bone mineral density $[3,4]$ demonstrate the importance of bone metabolism and bone turnover markers in these patients; so, we believe that osteocalcin and $\beta$-crosslaps should be included routinely in the mineral and metabolic tests performed to patients with recurrent calcium stones as they provide useful information on bone metabolic activity. Moreover, the ratio $\beta$-crosslaps/osteocalcin has been evaluated and is higher in patients with recurrent calcium stones [4]. This demonstrates that bone resorption is higher than bone formation, which is the most important reason for bone density loss in these patients.

\section{References}

1. Ghazali A, Bataille P, Solal MC, Marié A, Brazier M, Sebert JL, Prin L, Fournier A (1995) Bone involvement in idiopathic calcium lithiasis. Nephrologie 16:351-369

2. Lombardi G, Lanteri P, Colombini A, Banfi G (2012) Blood biochemical markers of bone turnover: pre-analytical and technical aspects of sample collection and handling. Clin Chem Lab Med 50:771-789

3. Arrabal-Polo MA, Arrabal-Martin M, de Haro-Munoz T, LopezLeon VM, Merino-Salas S, Ochoa-Hortal MA, Garrido-Gomez J, Lahoz-Garcia C, Zuluaga-Gomez A (2011) Mineral density and bone remodelling markers in patients with calcium lithiasis. BJU Int 108(11):1903-1908

4. Arrabal-Polo MA, Arrabal-Martin M, de Haro-Muñoz T, PoyatosAndujar A, Palao-Yago F, Zuluaga-Gomez A (2012) Biochemical determinants of severe lithogenic activity in patients with idiopathic calcium nephrolithiasis. Urology 79:48-54 